\title{
Supporting information Public LC-Orbitrap Tandem Mass Spectral Library for Metabolite Identification
}

\author{
Prasad Phapale $^{1^{*}}$, Andrew Palmer ${ }^{1 \#}$, Rose Muthoni Gathungu ${ }^{1}$, Dipali Kale ${ }^{4}$, Britta Brügger ${ }^{4}$, Theodore \\ Alexandrov ${ }^{1,2,3,{ }^{*}}$ \\ ${ }^{1}$ Metabolomics Core Facility, EMBL, Heidelberg, 69117 Germany; ${ }^{2}$ Structural and Computational Biology Unit, \\ European Molecular Biology Laboratory (EMBL), Heidelberg, 69117 Germany; ${ }^{3}$ Skaggs School of Pharmacy and \\ Pharmaceutical Sciences, University of California San Diego, CA 92093, La Jolla, USA; ${ }^{4}$ Heidelberg University \\ Biochemistry Center (BZH), Heidelberg, 69120 Germany \\ \#Present address: BASF SE, Ludwigshafen, 67063 Germany \\ *Correspondence: theodore.alexandrov@embl.de and prasad.phapale@embl.de
}

\section{Supplementary Methods}

Table-S1: LC-MS parameters for six protocols used for generating EMBL-MCF spectral library

Other LC parameters:

Q-exactive Orbitrap Mass spec Parameters

Data analysis for online upload and curation of the LC-MS/MS datasets

\section{Supplementary Figures and Tables}

Supplementary Table S2: Feature comparison for spectral libraries

Supplementary Figure S1: Separation of isomers Glucose 6-phosphate and Fructose 6-phosphate

Supplementary Figure S2) Fragmentation Spectrum layout view

Supplementary Figure S3a) Identification of Inosine from HCT116 cell extract by matching exact mass and RT with standard XIC from the library

Supplementary Figure S3b) Identification of Inosine from HCT116 cell extract

Supplementary Figure S4a) Identification of Uridine from HCT116 cell extract by matching exact mass and RT with standard XIC from the library

Supplementary Figure S4b) Identification of Uridine from HCT116 cell extract

Supplementary Figure S5a) Identification of Glutamine from HCT116 cell extract by matching exact mass and RT with standard XIC from the library. The false positive peak at 8.70 min was rejected.

Supplementary Figure S5b) Identification of Glutamine from HCT116 cell extract

Supplementary Figure S6a) Identification of Asparagine from HCT116 cell extract by matching exact mass and RT with standard XIC from the library. The false positive peak at $9.4 \mathrm{~min}$ was rejected.

Supplementary Figure S6b) Identification of Asparagine from HCT116 cell extract

Supplementary Figure S7) Curation of Malate peak: Although there is MS/MS match, the annotation of Malate was rejected due to poor peak shape.

Supplementary Table S3: List of metabolites analysed on amino, amide and zicHILIC columns for comparison of chromatographic performance.

Supplementary Figure S8) The comparison of amide, amine and zic-HILIC columns for amino acid analysis shows amide column as a good compromise between retention of polar metabolites, sensitivity, and analytical stability Supplementary Figure S9) Comparison of chromatographic performance of 3 columns for analysis of Creatinine standard

Supplementary Figure S10) Comparison of chromatographic performance of 3 columns for analysis of Tryptophan standard

Supplementary Figure S11) Comparison of chromatographic performance of 3 columns for analysis of Carnosine standard 
Supplementary Figure S12) Comparison of chromatographic performance of 3 columns for analysis of Glutamic acid standard

SUPPLEMENTARY EXCEL DATA FILES (ATTACHED SEPARATELY):

Supplementary Information_IROA MSMLS kit

Supplementary Information_Unique IDs 


\section{Supplementary Methods}

Table-S1: LC-MS parameters for six protocols used for generating EMBL-MCF spectral library

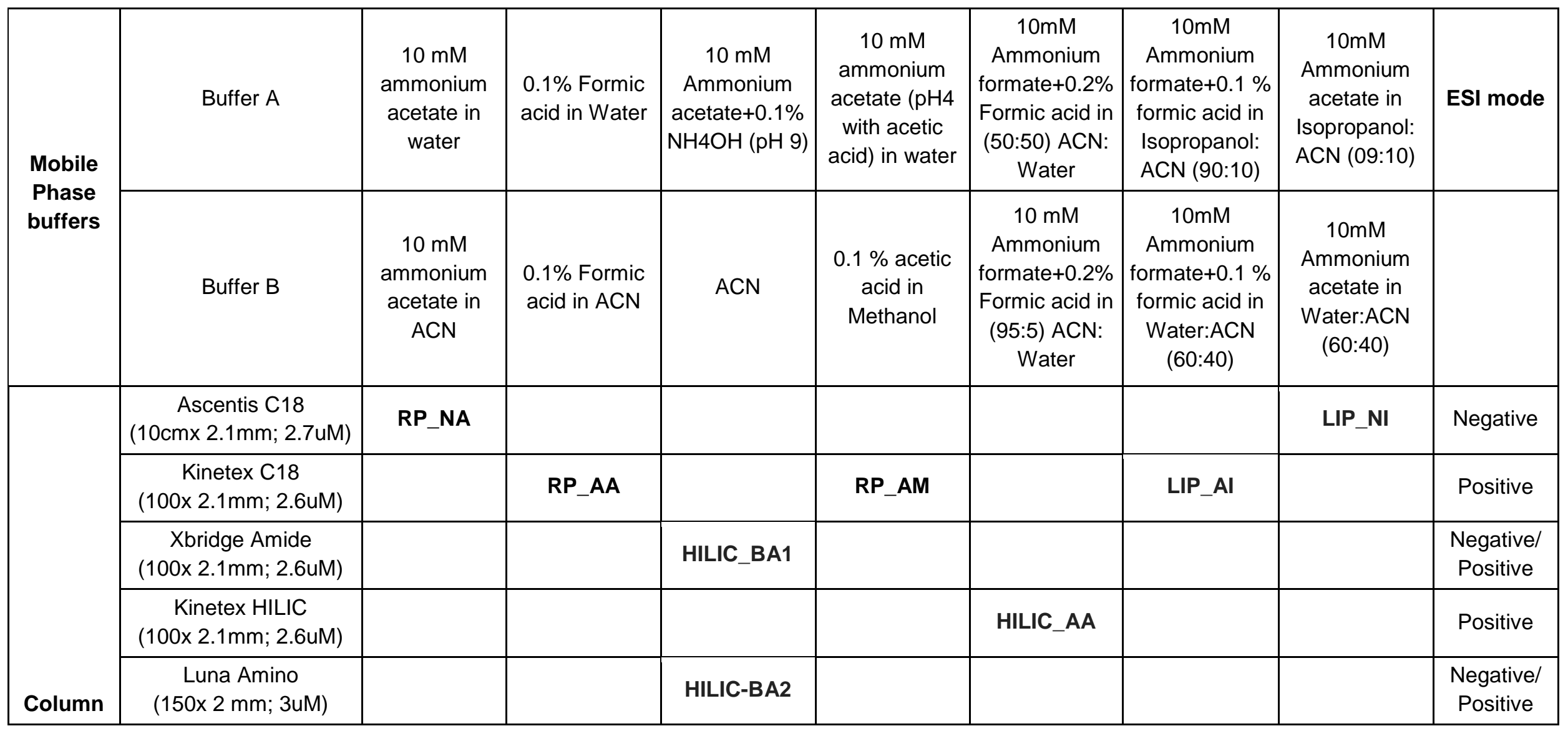




\section{Other LC parameters:}

RP_AA:

Column Temperature: $30^{\circ} \mathrm{C}$

Flow: $0.3 \mathrm{ml} / \mathrm{min}$

Gradients:

\begin{tabular}{|c|c|}
\hline Time & \% Buffer B \\
\hline 0 & 95 \\
\hline 3 & 95 \\
\hline 18 & 25 \\
\hline 23 & 2 \\
\hline 30 & 2 \\
\hline 31 & 95 \\
\hline 35 & 95 \\
\hline
\end{tabular}

RP_NA:

Column Temperature: $30^{\circ} \mathrm{C}$

Flow: $0.26 \mathrm{ml} / \mathrm{min}$

Gradients:

\begin{tabular}{|l|l|}
\hline Time & \% Buffer B \\
\hline 0 & 95 \\
\hline 3 & 95 \\
\hline 18 & 25 \\
\hline 23 & 2 \\
\hline 30 & 2 \\
\hline 31 & 95 \\
\hline 35 & 95 \\
\hline
\end{tabular}

\section{RP_AM:}

Column Temperature: $30^{\circ} \mathrm{C}$

Flow: $0.3 \mathrm{ml} / \mathrm{min}$

Gradients:

\begin{tabular}{|l|l|}
\hline Time & \% Buffer B \\
\hline 0 & 5 \\
\hline 1 & 5 \\
\hline 7 & 54 \\
\hline 8 & 90 \\
\hline 11 & 90 \\
\hline 11.5 & 5 \\
\hline 14 & 5 \\
\hline
\end{tabular}




\section{HILIC BA:}

Column Temperature: $30^{\circ} \mathrm{C}$

Flow: $0.26 \mathrm{ml} / \mathrm{min}$

Gradients:

\begin{tabular}{|l|l|}
\hline Time & \% Buffer B \\
\hline 0 & 85 \\
\hline 2 & 85 \\
\hline 12 & 10 \\
\hline 14 & 10 \\
\hline 14.1 & 85 \\
\hline 16 & 85 \\
\hline
\end{tabular}

HILIC_AA:

Column Temperature: $30^{\circ} \mathrm{C}$

Flow: $0.2 \mathrm{ml} / \mathrm{min}$

Gradients:

\begin{tabular}{|l|l|}
\hline Time & \% Buffer B \\
\hline 0 & 100 \\
\hline 5 & 100 \\
\hline 11 & 30 \\
\hline 14 & 30 \\
\hline 15 & 100 \\
\hline 18 & 100 \\
\hline
\end{tabular}

LIP_Al:

Column Temperature: $30^{\circ} \mathrm{C}$

Flow: $0.26 \mathrm{ml} / \mathrm{min}$

Gradients:

\begin{tabular}{|l|l|}
\hline Time & \% Buffer B \\
\hline 0 & 20 \\
\hline 0.5 & 20 \\
\hline 3 & 50 \\
\hline 10 & 70 \\
\hline 18 & 97 \\
\hline 22 & 97 \\
\hline 22.1 & 20 \\
\hline 25 & 20 \\
\hline
\end{tabular}




\section{LIP_NI:}

Column Temperature: $30^{\circ} \mathrm{C}$

Flow: $0.26 \mathrm{ml} / \mathrm{min}$

Gradients:

\begin{tabular}{|l|l|}
\hline Time & \% Buffer B \\
\hline 0 & 32 \\
\hline 1.5 & 32 \\
\hline 4 & 45 \\
\hline 5 & 52 \\
\hline 8 & 58 \\
\hline 11 & 66 \\
\hline 14 & 70 \\
\hline 18 & 75 \\
\hline 21 & 97 \\
\hline 25 & 97 \\
\hline 25.1 & 32 \\
\hline 28 & 32 \\
\hline
\end{tabular}




\section{Q-exactive Orbitrap Mass spec Parameters}

Molecules were detected with HRMS full scan at the mass resolving power $\mathrm{R}=70000$ in the mass range of $60-900 \mathrm{~m} / \mathrm{z}$ (for small molecules) or 150 to $1500 \mathrm{~m} / \mathrm{z}$ (for lipids). The data-dependent (DDA) MS/MS scans for 10 most intense (TOP10)were obtained along with full scans using higherenergy collisional dissociation (HCD) of normalized collision energies (NCE) of 20, 40, and 60 units at the mass resolving power $R=17500$. The MS parameters in the Tune software (Thermo Scientific) were set as; spray voltage of $4.2 \mathrm{kV}$ (for negative mode $3.5 \mathrm{kV}$ ), sheath gas 35 , and auxiliary gas 5 units, S-Lens $65 \mathrm{eV}$, capillary temperature $350^{\circ} \mathrm{C}$, and vaporization temperature of auxiliary gas was set at $300^{\circ} \mathrm{C}$.

\section{Data analysis for online upload and curation of the LC-MS/MS datasets}

All acquired LC-MS/MS .raw files were converted to centroid .mzml format using Proteowizard software. A .mzml file for standard mixtures was then uploaded on curatr web-application and respective standards were selected from curatr database inventory

(http://curatr.mcf.embl.de/inventory/). The other parameters and metadata relevant to the data file were selected as:

1. Name of the standard (or MCF ID)

2. Expected adduct (based on LC-MS method used)

3. Anticipated MS accuracy for precursor (default value $10 \mathrm{ppm}$ )

4. Precursor quadrupole window (default value $1 \mathrm{Da}$ )

5. LC method information

6. MS method information

7. ESI ionization mode

8. MS ion analyzer used (default value QFT)

The parameters 1 to 4 are used by curatr software for extracting expected precursors, their adducts, and MS/MS spectra from a dataset. The input information 4 to 8 is stored with each dataset and spectra as LC-MS metadata. 


\section{Supplementary Figures and Tables}

Supplementary Table S2: Feature comparison for spectral libraries

\begin{tabular}{|l|l|l|l|}
\hline Library & LC info & Open access & Orbitrap \\
\hline mzCloud & $x$ & - & $\checkmark$ \\
\hline NIST14 & $\checkmark \square$ & $x$ & - \\
\hline Metlin & $x$ & - & $x$ \\
\hline Massbank/MoNa & - & $\checkmark$ & - \\
\hline GNPS & $x$ & $\checkmark$ & - \\
\hline HMDB & $x$ & $\checkmark$ & $x$ \\
\hline $\begin{array}{l}\text { EMSL } \\
\text { (this library) }\end{array}$ & $\checkmark \square$ & $\checkmark$ & $\checkmark$ \\
\hline
\end{tabular}


Supplementary Figure S1: Separation of isomers Glucose 6-phosphate and Fructose 6-phosphate

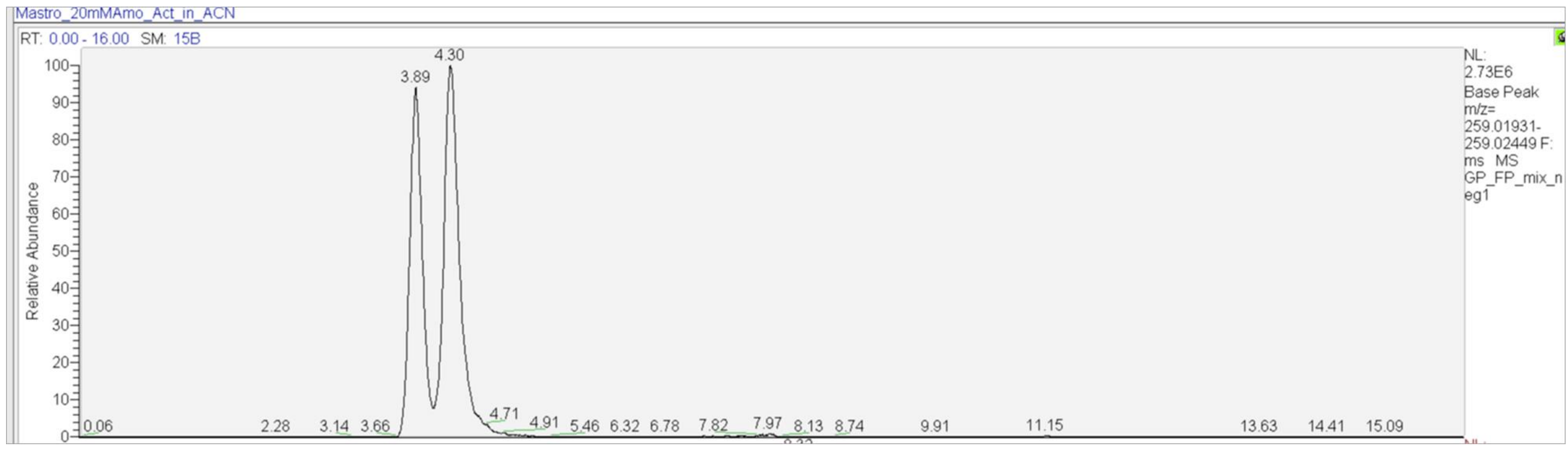

\section{Column: C18 Shimadzu Mastro column}

Mobile phase-A: $20 \mathrm{mM}$ ammonium acetate

Mobile phase-B: Acetonitrile

\section{ESI negative mode}

Other parameters are same as RP_NA method (Supplementary Table S1) 


\section{Supplementary Figure S2) Fragmentation Spectrum layout view}

Fragmentation Spectrum for 44: Guanosine, [M-H]

Scan number: 1277

Precursor $\mathrm{m} / z: 282.08420$

Retention time: $3.38 \mathrm{~min}$

Collision energy: collision energy 30.0 electronvolt

Raw data: Std_ADP_phos_mix_170117112458.mzML.

Splash: calculating.

LC Method: Column: Xbridge Amide (100X 2.1mm; 2.6uM); Temperature: 30 C; Mobile phase A: $7.5 \mathrm{~mm} \mathrm{Ammonium} \mathrm{acetate+0.1 \%} \mathrm{NH4OH,} \mathrm{Mobile} \mathrm{phase} \mathrm{B:} \mathrm{ACN;} \mathrm{Flow:} \mathrm{0.3} \mathrm{m/min} \mathrm{Gradient:} \mathrm{(Time/ \%} \mathrm{B:} \mathrm{0/} \mathrm{85,} \mathrm{2/} \mathrm{85,} \mathrm{12/} \mathrm{10,} \mathrm{14/} \mathrm{10,} \mathrm{14.1/} \mathrm{85,} \mathrm{16/} \mathrm{85)}$

MS Method: ESI_Neg

Review Information

Precursor intensity: 267338

Precursor Fraction: 1.0

Date added: April 27, 2017

Date curated: April 28, 2017

Curator: prasad

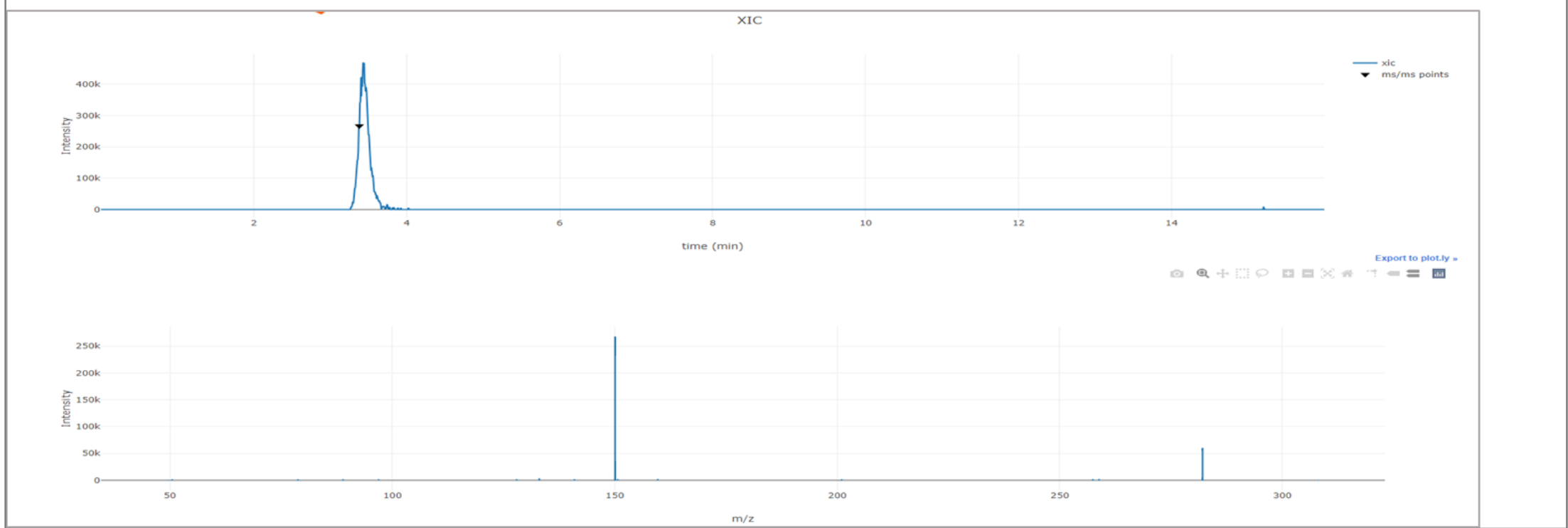


Supplementary Figure S3a) Identification of Inosine from HCT116 cell extract by matching exact mass and RT with standard XIC from the library
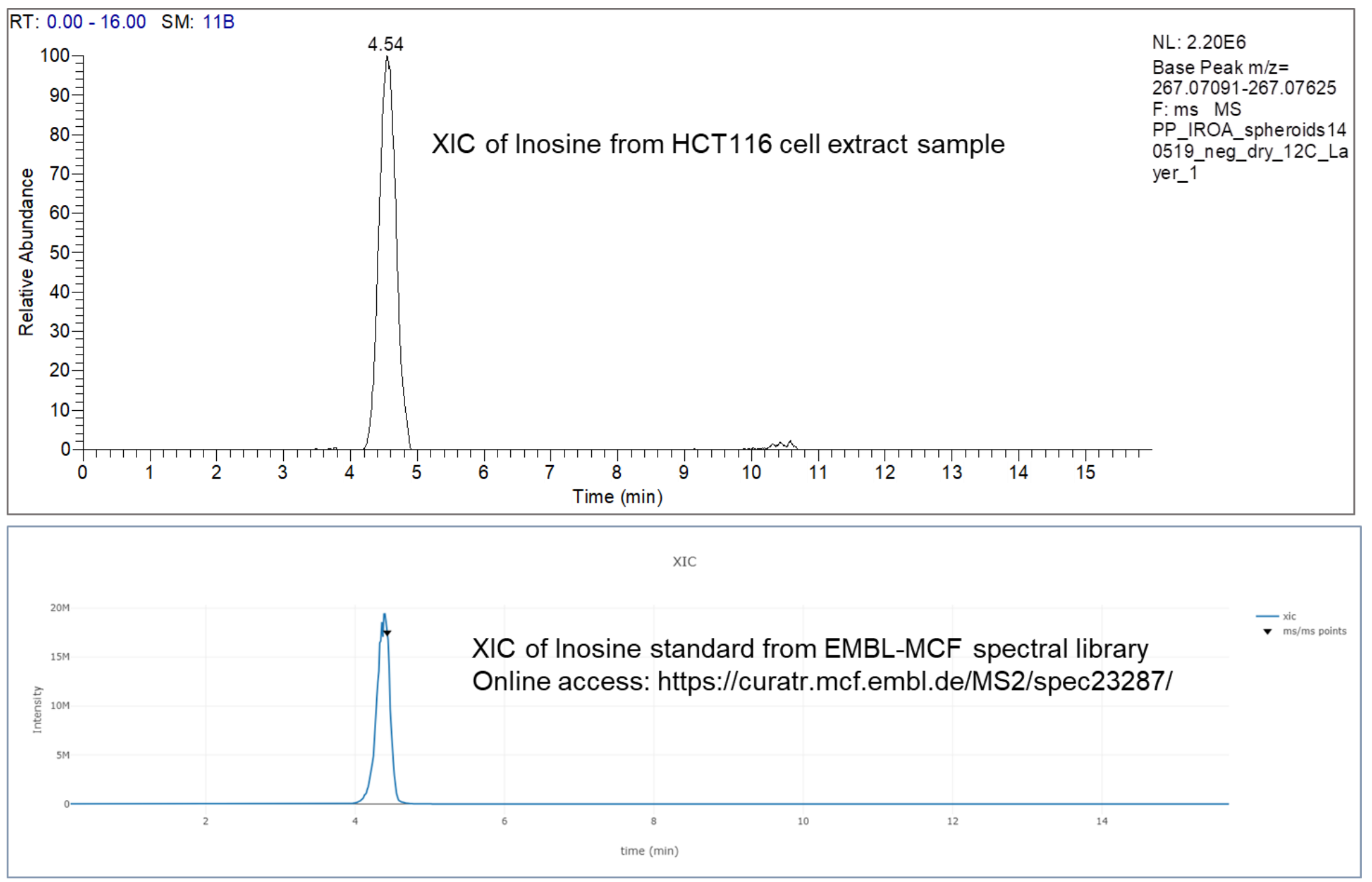
Supplementary Figure S3b) Identification of Inosine from HCT116 cell extract

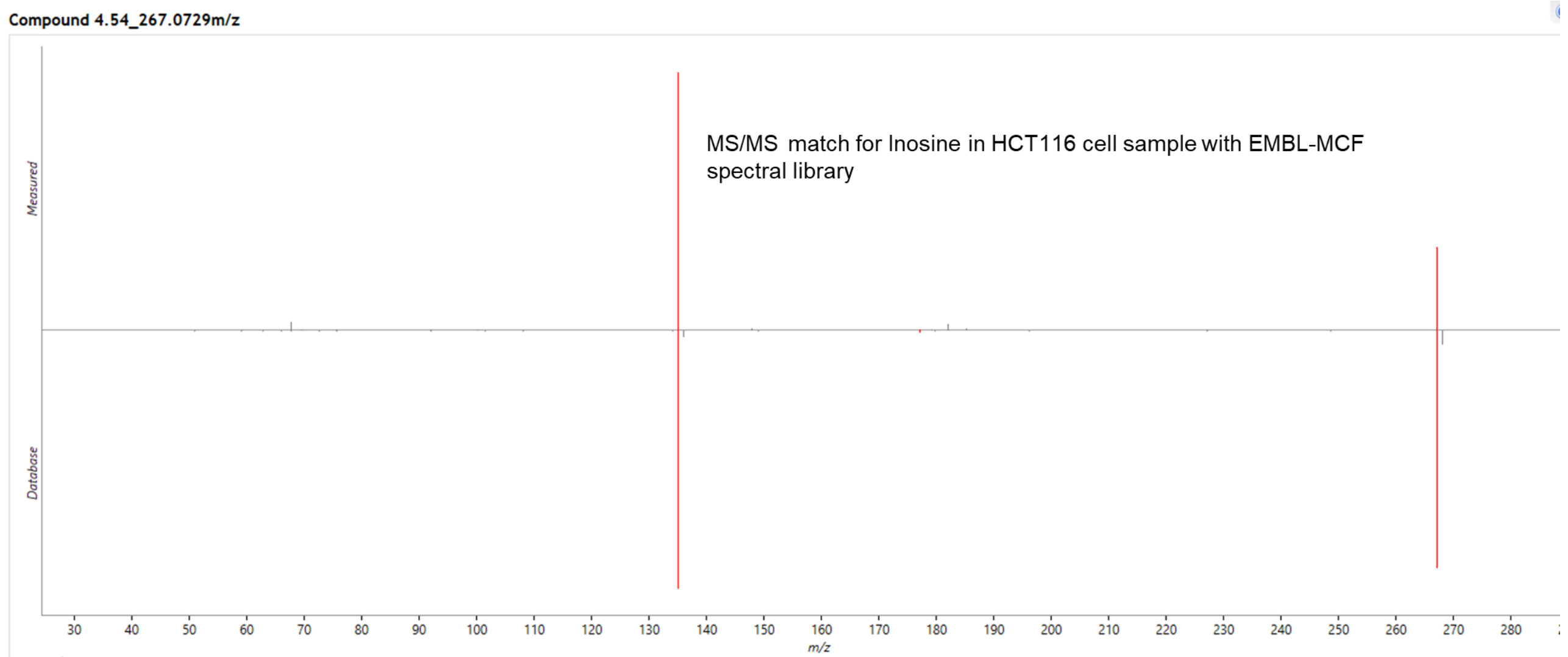

Legend: Matched fragment Unmatched fragment 
Supplementary Figure S4a) Identification of Uridine from HCT116 cell extract by matching exact mass and RT with standard XIC from the library

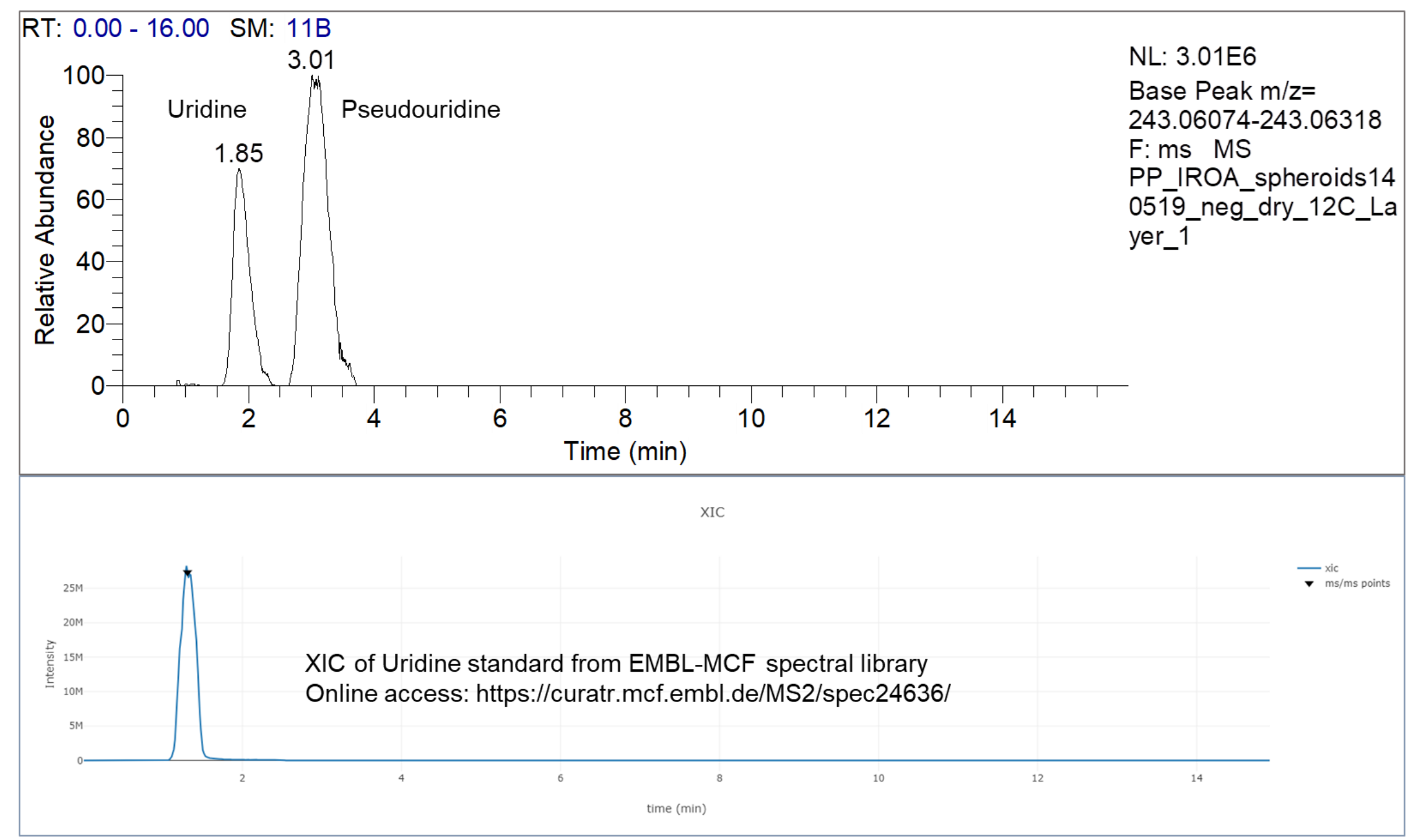




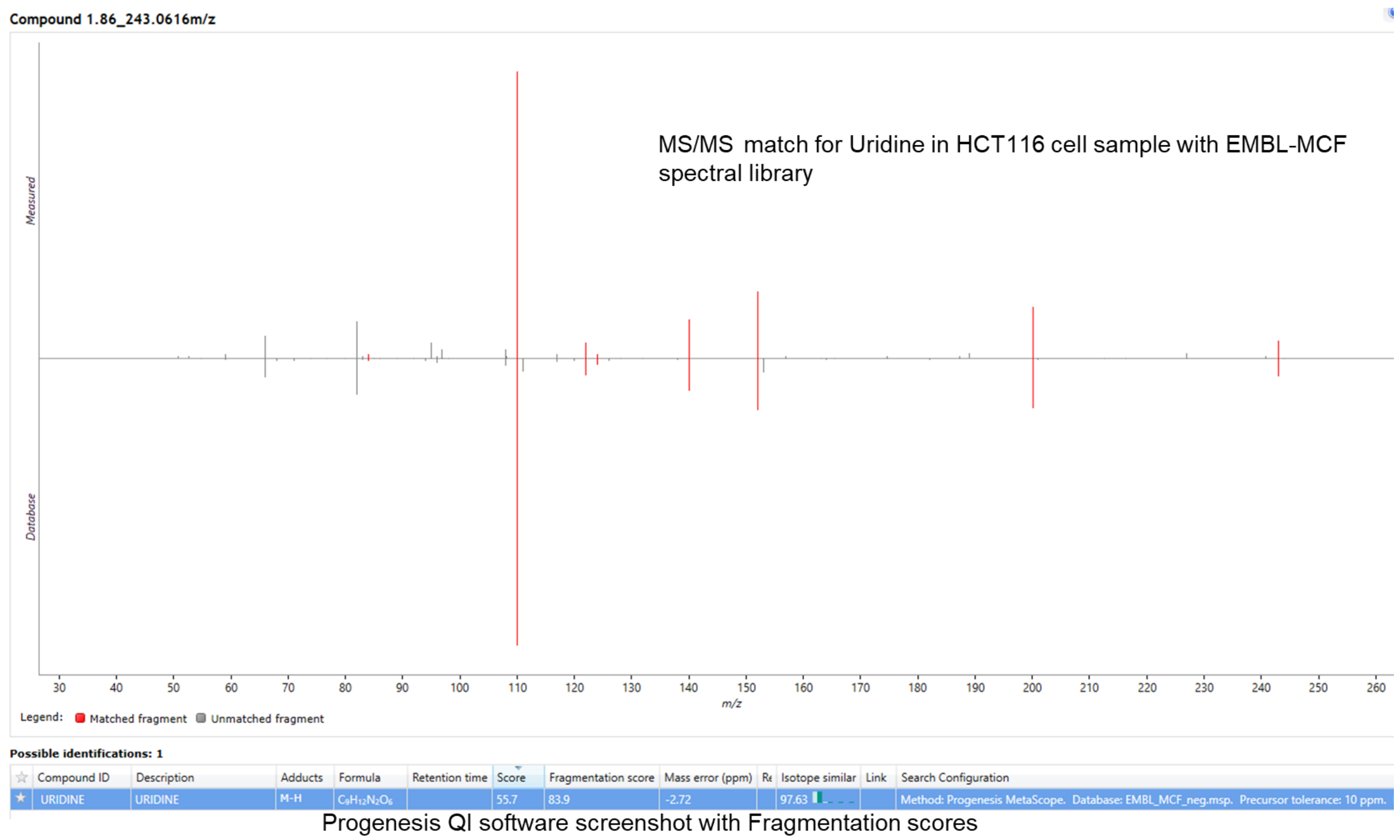


Supplementary Figure S5a) Identification of Glutamine from HCT116 cell extract by matching exact mass and RT with standard XIC from the library. The false positive peak at 8.70 min was rejected.

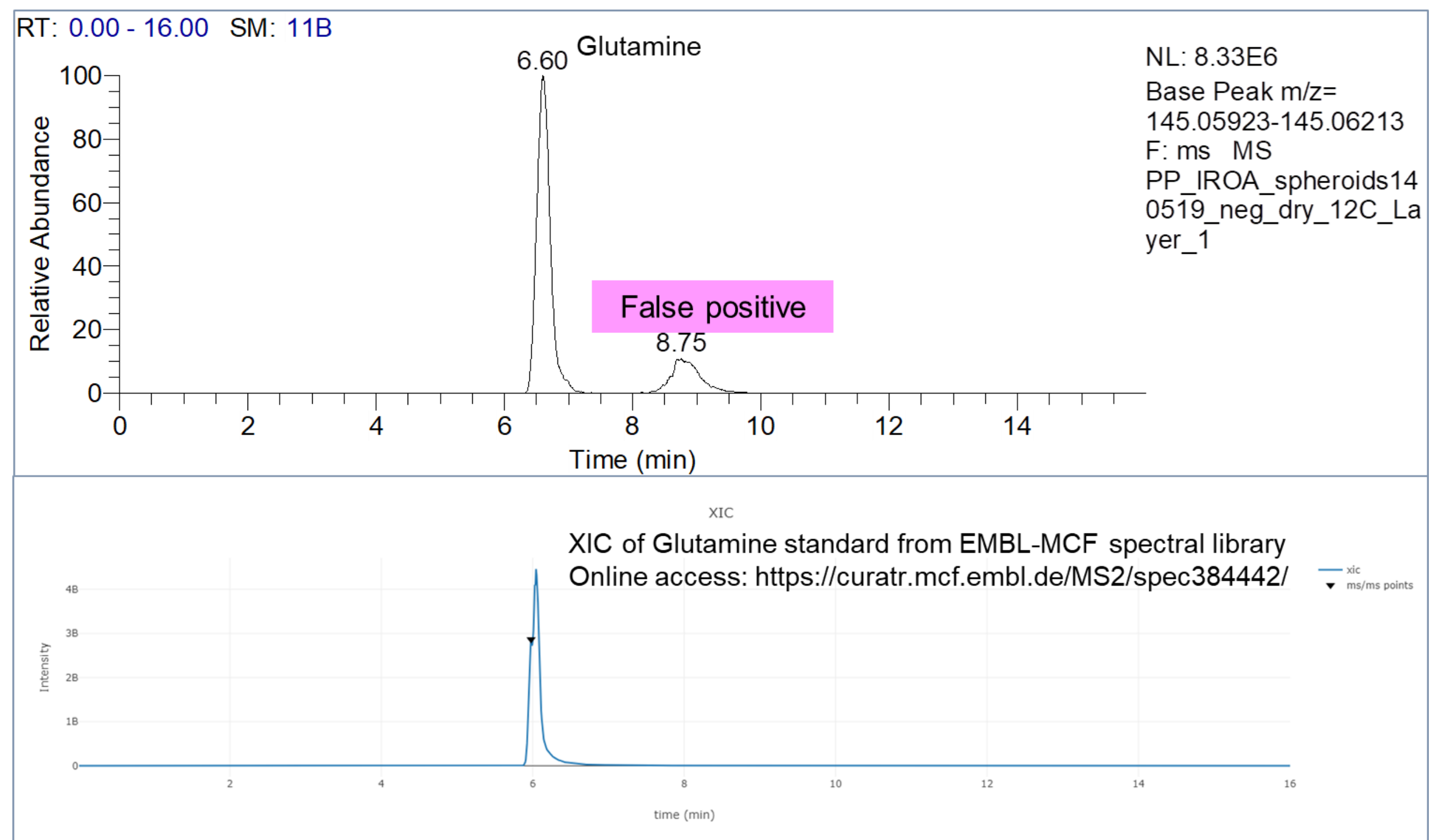




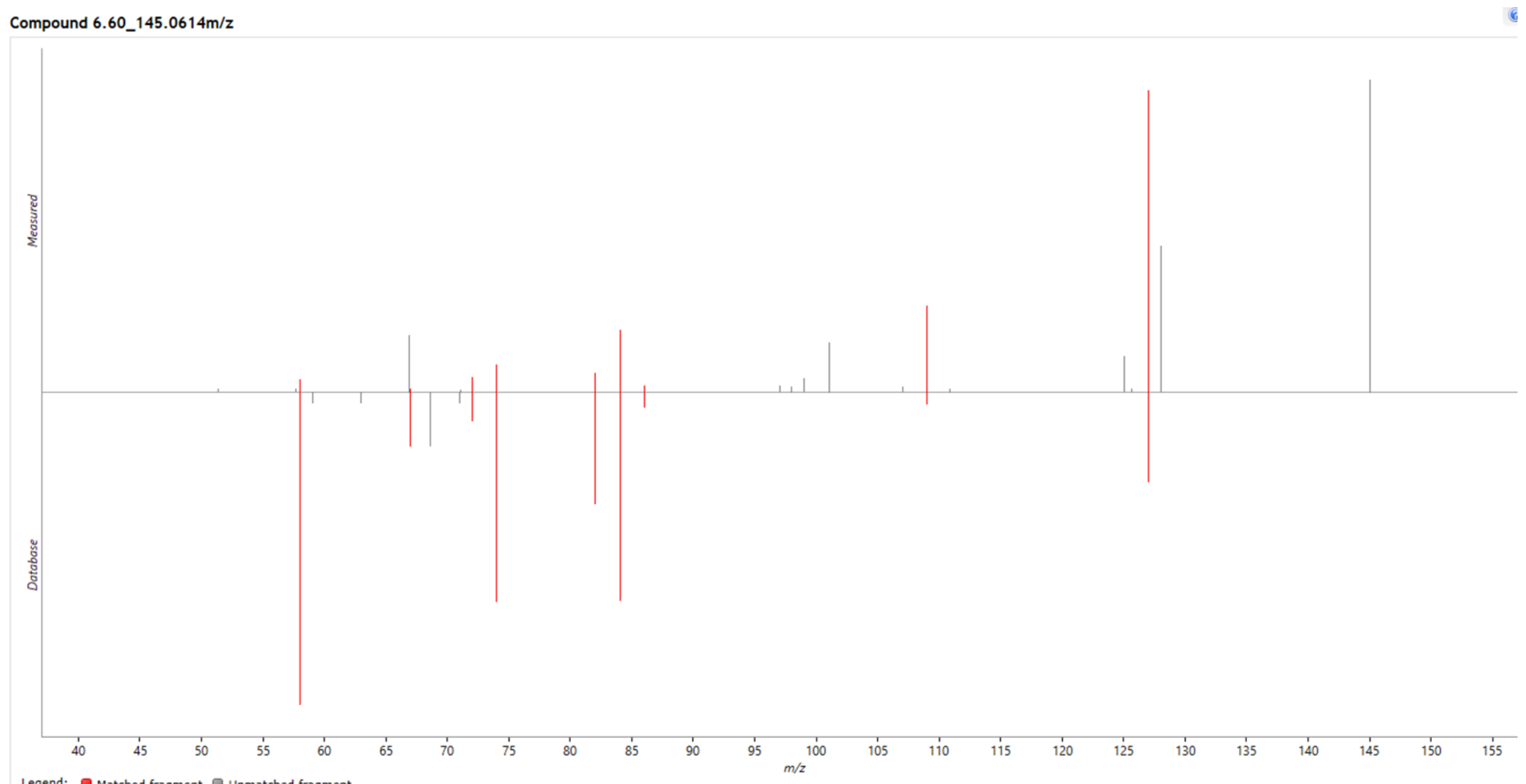

Legend: Matched fragment 0 Unmatched fragment

Progenesis QI software screenshot with Fragmentation scores 
Supplementary Figure S6a) Identification of Asparagine from HCT116 cell extract by matching exact mass and RT with standard XIC from the library. The false positive peak at 9.4 min was rejected.

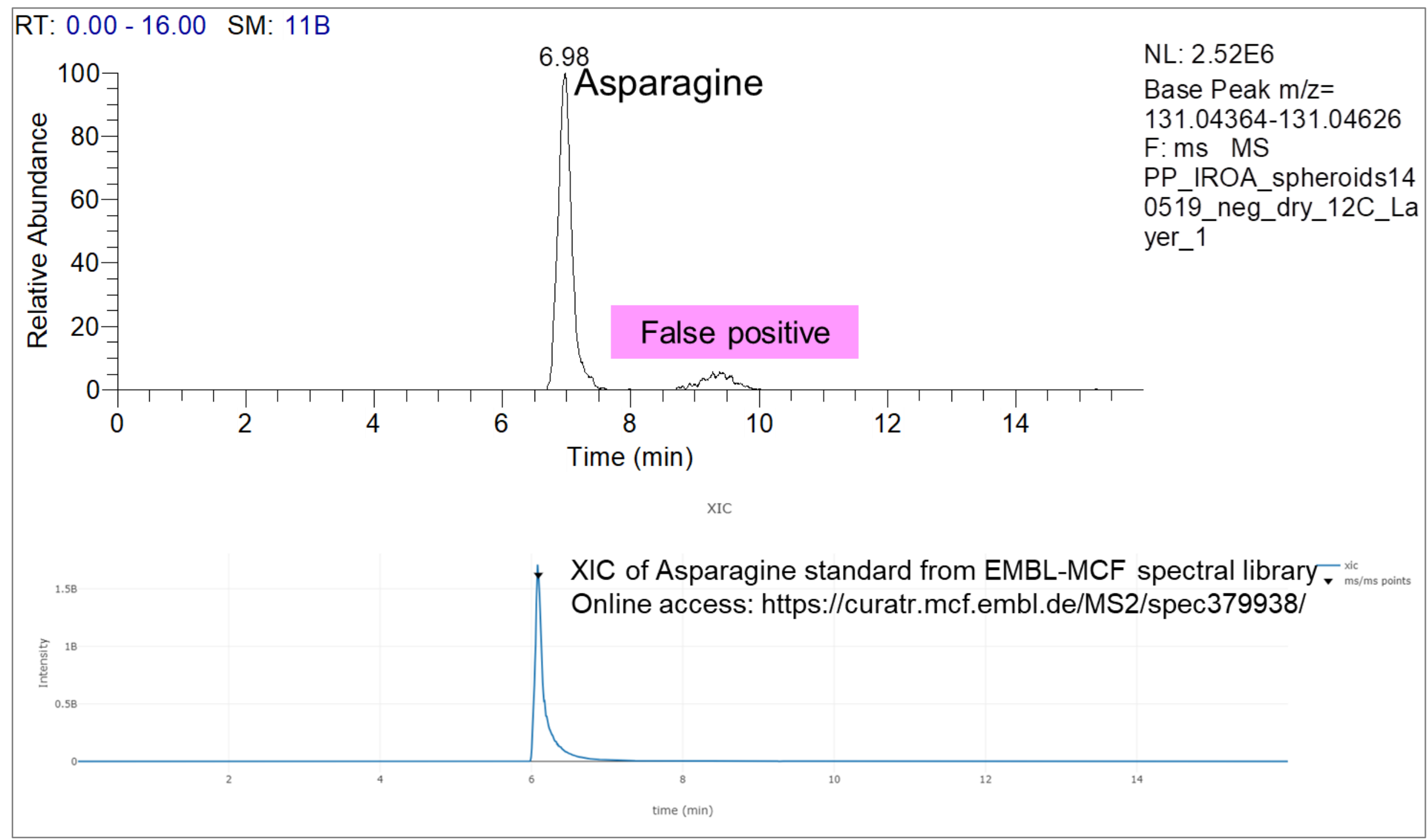


Supplementary Figure S6b) Identification of Asparagine from HCT116 cell extract

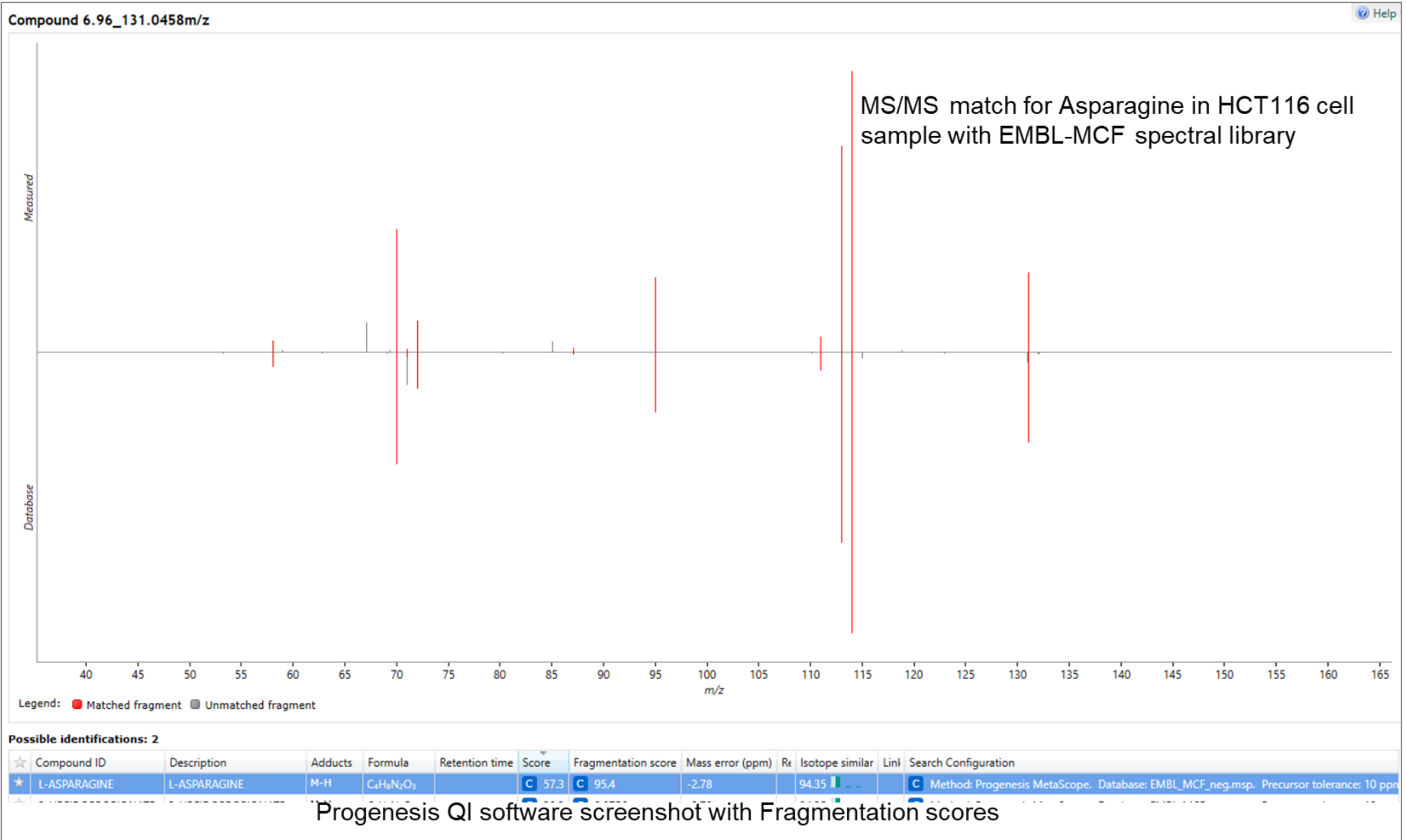


Supplementary Figure S7) Curation of Malate peak: Although there is MS/MS match, the annotation of Malate was rejected due to poor peak shape.

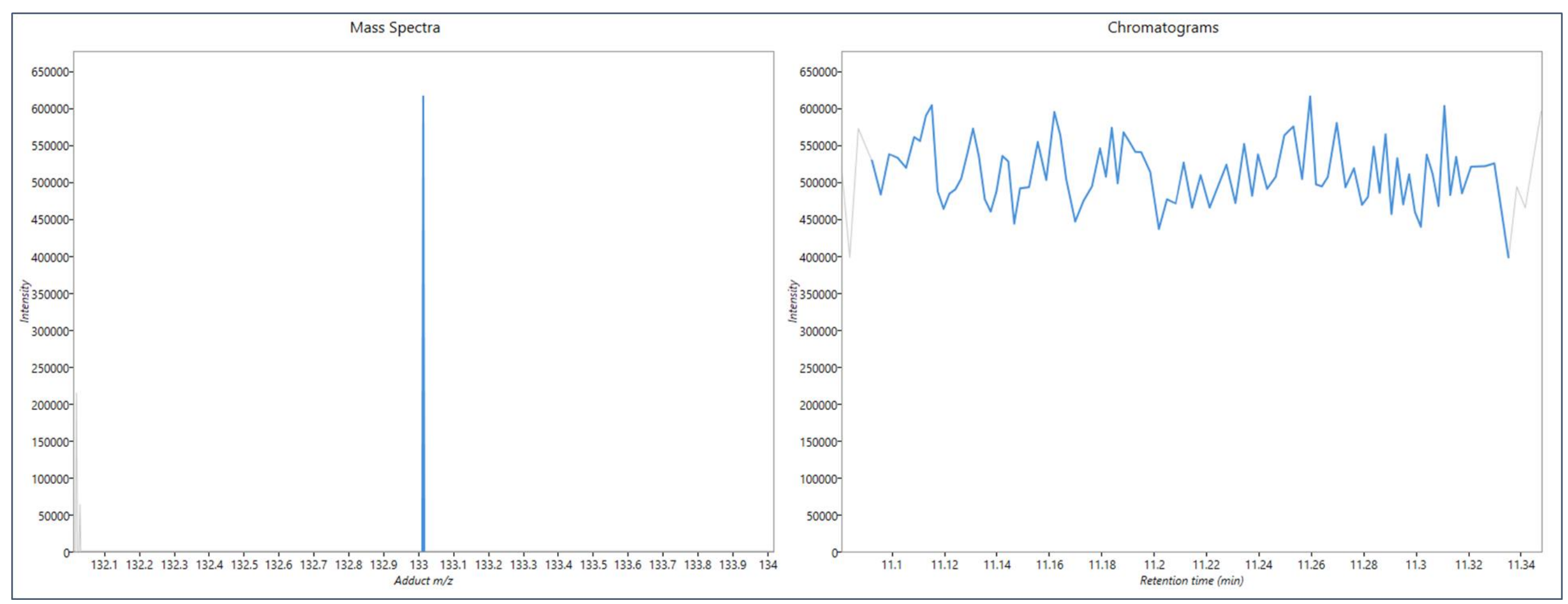


Supplementary Table S3: List of metabolites analysed on amino, amide and zicHILIC columns for comparison of chromatographic performance.
1. Glycine
2. Alanine
3. Sarcosine
4. Amino-n-butyric acid
5. Serine
6. Creatinine
7. Proline
8. Valine
9. Threonine
10. Taurine
11. Hydroxy-proline
12. Leucine
13. Ornithine
14. Aspartic acid
15. Homocysteine
16. Lysine
17. Glutamic acid
18. Methionine
19. Histidine
20. Aminoadipic
21. Hydroxylysine
22. Phenylalanine
23. Methyl histidine
24. Arginine
25. Citrulline
26. Tyrosine
27. Tryptophan
28. Cystathionine
29. Carnosine
30. Cvstine 
Supplementary Figure S8) The comparison of amide, amine and zic-HILIC columns for amino acid analysis shows amide column as a good compromise between retention of polar metabolites, sensitivity, and analytical stability

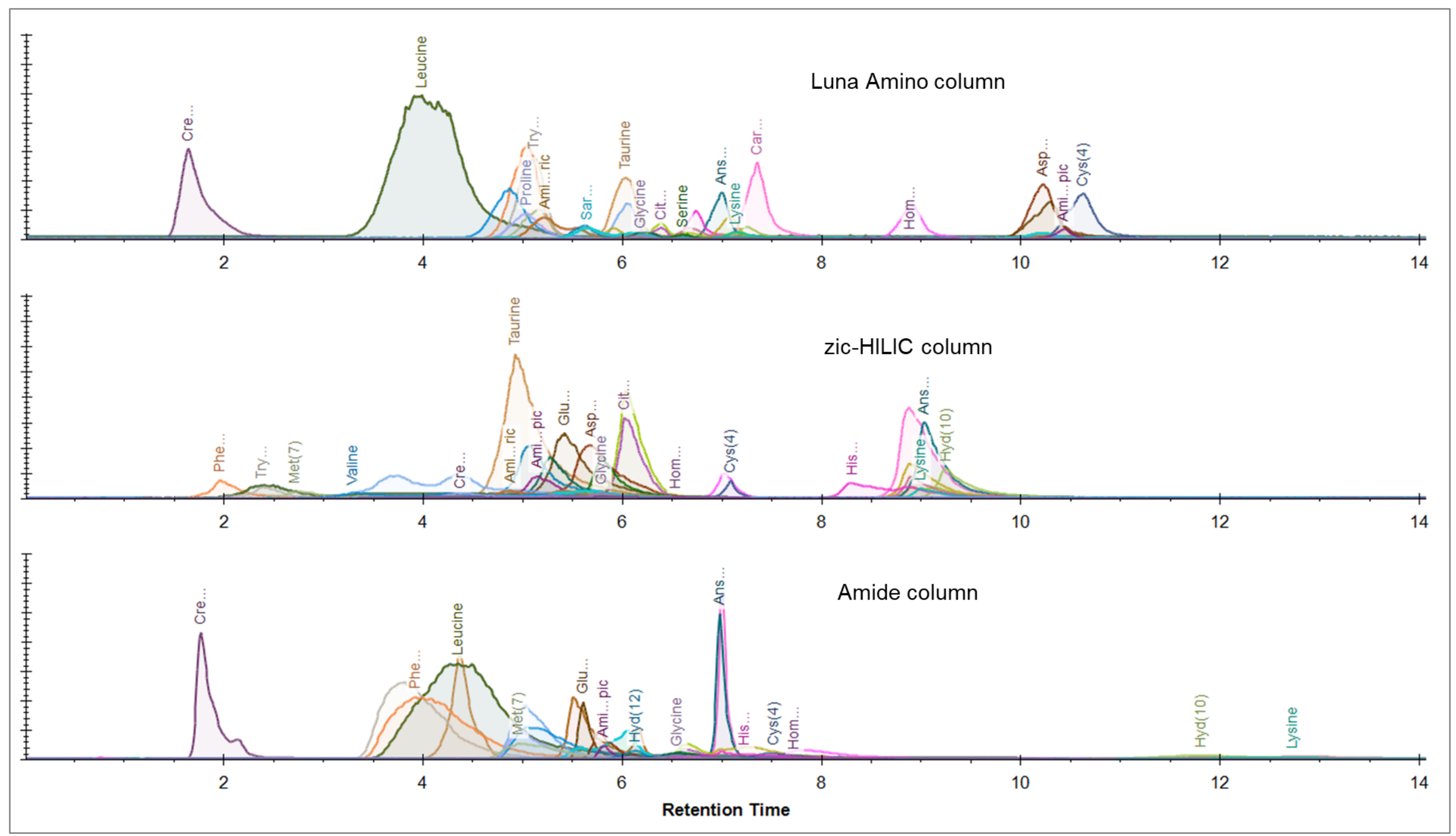


Supplementary Figure S9) Comparison of chromatographic performance of 3 columns for analysis of Creatinine standard

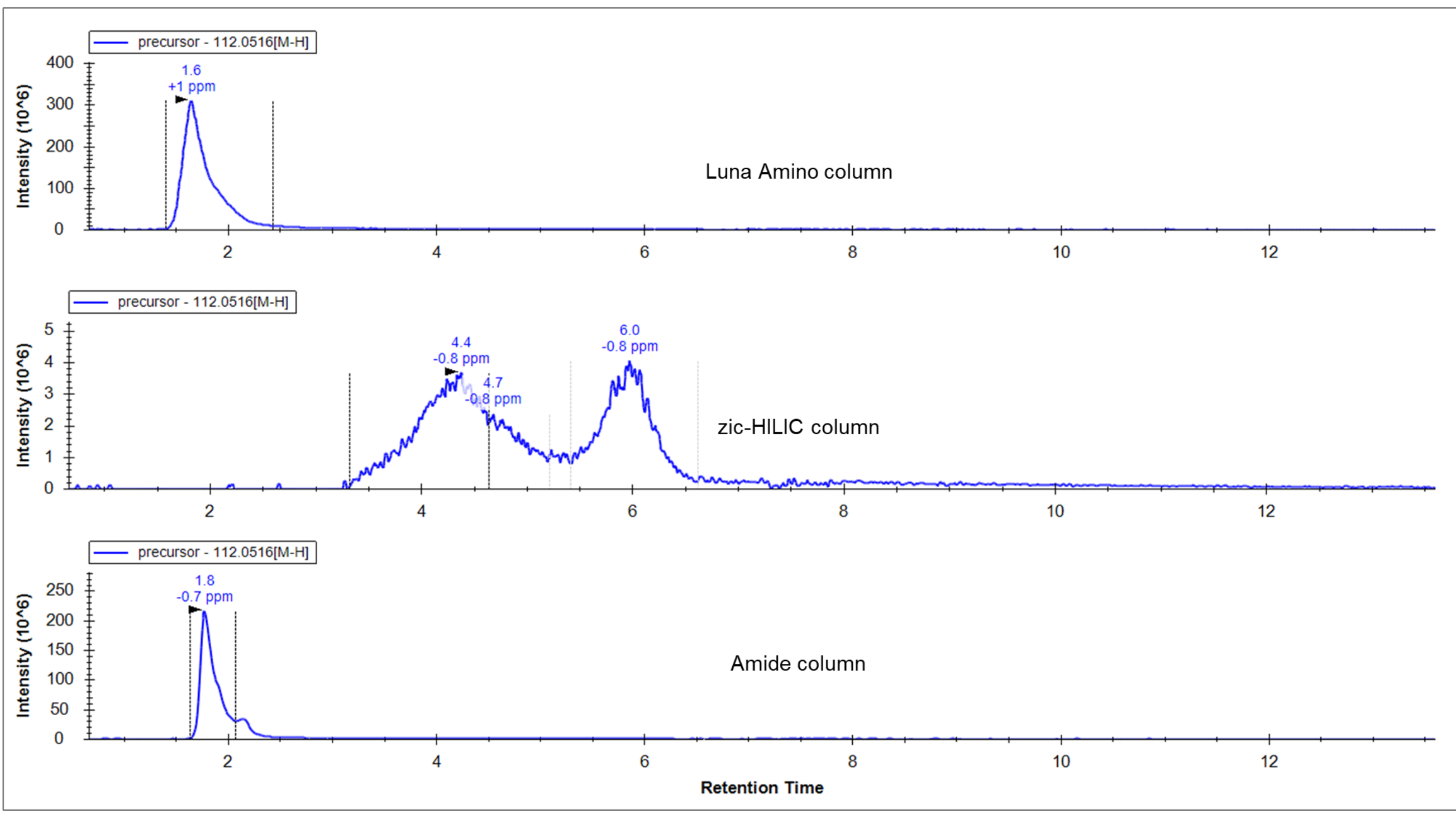


Supplementary Figure S10) Comparison of chromatographic performance of 3 columns for analysis of Tryptophan standard

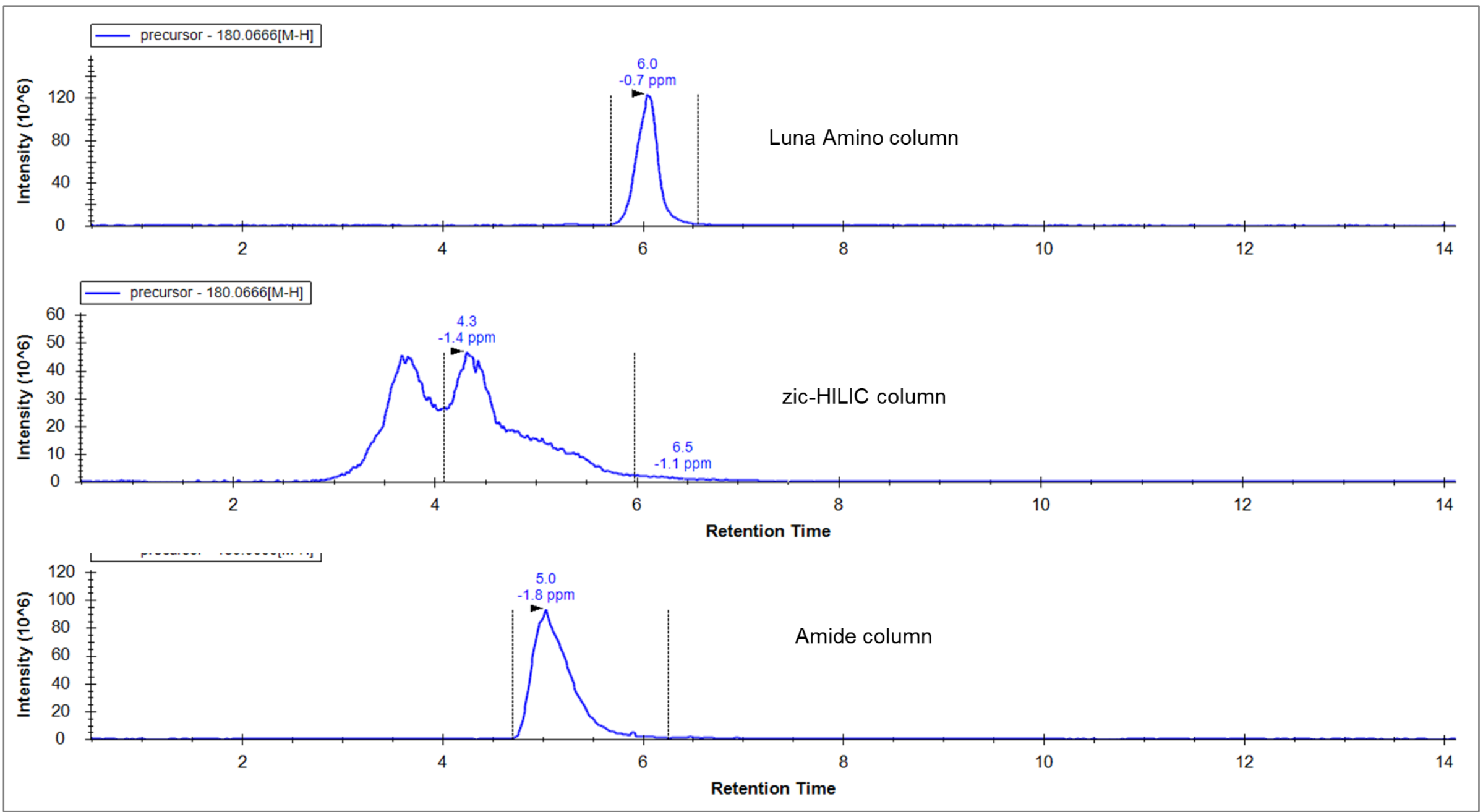


Supplementary Figure S11) Comparison of chromatographic performance of 3 columns for analysis of Carnosine standard

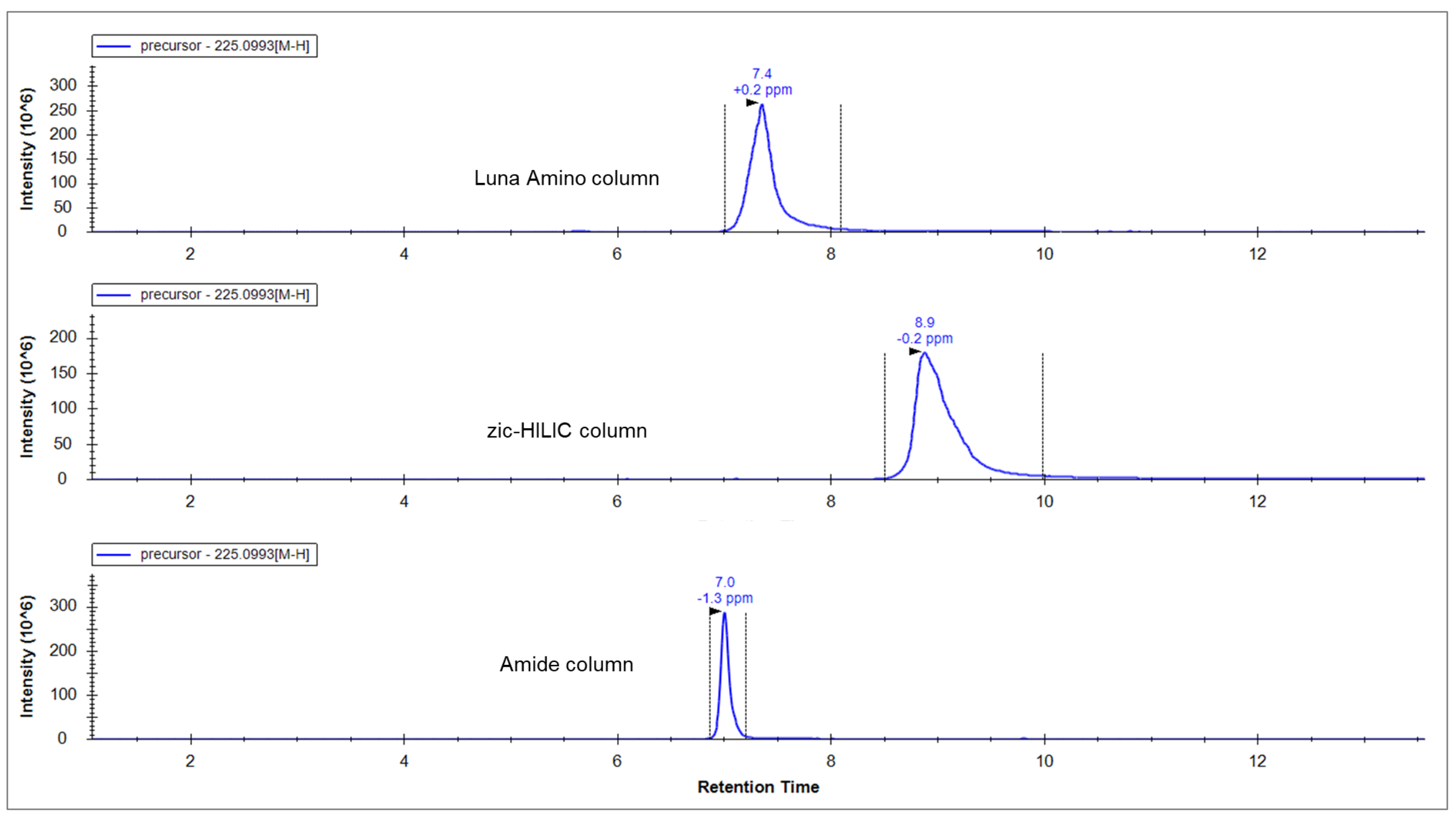


Supplementary Figure S12) Comparison of chromatographic performance of 3 columns for analysis of Glutamic acid standard

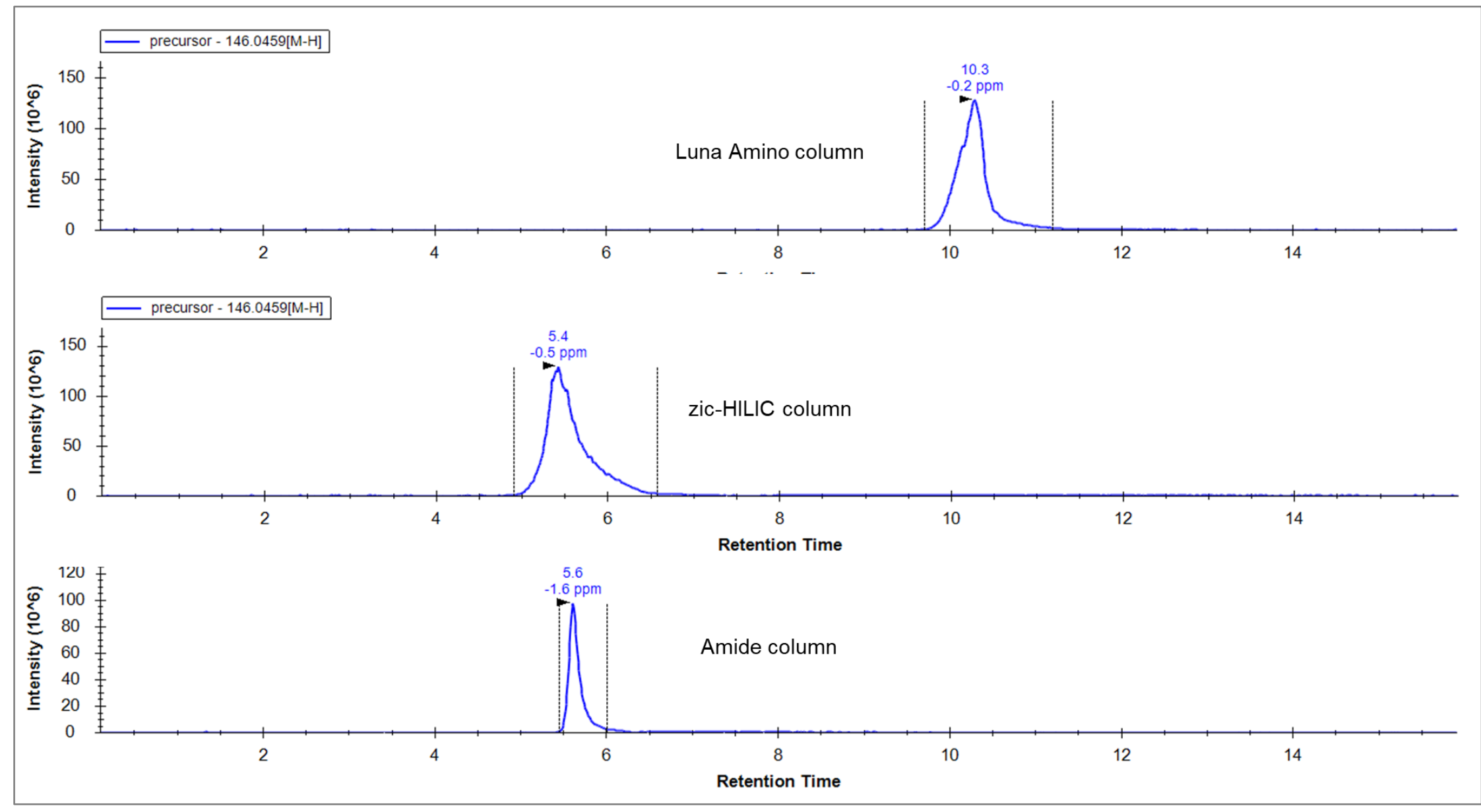




\section{Supplementary excel data files (attached separately):}

Supplementary Information_IROA MSMLS kit

Supplementary Information_Unique IDs 